\title{
Schwinger mechanism in electromagnetic field in de Sitter spacetime
}

\author{
Ehsan Bavarsad ${ }^{1, \star}$, Sang Pyo Kim ${ }^{2,3}$, Clément Stahl ${ }^{4}$, and She-Sheng Xue ${ }^{5,6}$ \\ ${ }^{1}$ Department of Physics, University of Kashan, 8731753153, Kashan, Iran \\ ${ }^{2}$ Department of Physics, Kunsan National University, Kunsan 54150, Korea \\ ${ }^{3}$ Center for Relativistic Laser Science, Institute for Basic Science, Gwangju 61005, Korea \\ ${ }^{4}$ Instituto de Física, Pontificia Universidad Católica de Valparaíso, Casilla 4950, Valparaíso, Chile \\ ${ }^{5}$ ICRANet, Piazzale della Repubblica 10, 65122 Pescara, Italy \\ ${ }^{6}$ Dipartimento di Fisica, Universita di Roma "La Sapienza", Piazzale Aldo Moro 5, 00185 Rome, Italy
}

\begin{abstract}
We investigate Schwinger scalar pair production in a constant electromagnetic field in de Sitter (dS) spacetime. We obtain the pair production rate, which agrees with the Hawking radiation in the limit of zero electric field in dS. The result describes how a cosmic magnetic field affects the pair production rate. In addition, using a numerical method we study the effect of the magnetic field on the induced current. We find that in the strong electromagnetic field the current has a linear response to the electric and magnetic fields, while in the infrared regime, is inversely proportional to the electric field and leads to infrared hyperconductivity.
\end{abstract}

\section{Introduction}

The Schwinger effect [1] is a phenomenon of pair production due to a strong electromagnetic field background. Since the early universe scenarios predict the existence of strong electromagnetic fields, it is natural to consider the Schwinger effect in this context. The pioneering works studied this effect in the 1+1 dimensional [2] and 1+3 dimensional [3] de Sitter spacetimes. To describe Schwinger effect in a curved spacetime, the more relevant quantity is the induced current $[2,3]$. Indeed, it is not plagued by the notion of particle in the adiabatic future, which allows one to explore a broader parameter space. In [4], the Schwinger mechanism in 1+2 dimensions was explored as an example of odd dimensional field theory in $\mathrm{dS}$.

In this proceedings' paper, we present some results of [5] and further elaborate the numerical results. The object of this work is to study the effect of a constant magnetic field background to the already present $\mathrm{dS}$ and electric backgrounds. This is a common generalization in flat spacetime where the analytic results are known for long [1], but it has never been investigated properly in dS. The origin of the observed large scale magnetic field is now an open question in cosmology but two main scenarios are emerging: an origin after recombination or a primordial one; for a recent review see, e.g., [6]. The effect of a magnetic field background on the scalar pair production in the spatially flat Friedmann-Lemaitre-Robertson-Walker type universes has been investigated in [7]. The perturbative amplitude for the fermion pair production in a magnetic field in dS has also been computed in [8].

\footnotetext{
^e-mail: bavarsad@kashanu.ac.ir
} 


\section{Schwinger Effect}

In order to analyze the Schwinger effect, we assume that the complex scalar field is a test field probing two background fields: the gravitational field described by the dS metric

$$
d s^{2}=\Omega^{2}(\tau)\left(d \tau^{2}-d x^{2}-d y^{2}-d z^{2}\right), \quad \Omega(\tau)=-\frac{1}{H \tau}, \quad \tau \in(-\infty, 0), \quad(x, y, z) \in \mathbb{R}^{3},
$$

where $H$ is the Hubble constant, and the electromagnetic field

$$
A_{\mu}(x)=-\frac{E}{H^{2} \tau} \delta_{\mu}^{3}+B y \delta_{\mu}^{1},
$$

where $E$ and $B$ are constants. The vector potential (2) leads to a constant electric and magnetic field parallel to each other in the conformal metric (1). The Schwinger pair production rate is obtained from the Bogoliubov coefficients. For a state with the comoving momentum $k$ and the Landau level $n$, the Bogoliubov coefficients are given by

$$
\alpha=\frac{(2|\gamma|)^{\frac{1}{2}} \Gamma(2 \gamma)}{\Gamma\left(\frac{1}{2}+\kappa+\gamma\right)} e^{\frac{i \pi}{2}(\kappa-\gamma)}, \quad \beta=-i \frac{(2|\gamma|)^{\frac{1}{2}} \Gamma(-2 \gamma)}{\Gamma\left(\frac{1}{2}+\kappa-\gamma\right)} e^{\frac{i \pi}{2}(\kappa+\gamma)},
$$

where the parameters have been defined as

$$
\begin{array}{lll}
k=\sqrt{k_{z}^{2}+(2 n+1) e B}, & \mu=\frac{m}{H}, & \lambda=\frac{e E}{H^{2}}, \\
\kappa=\frac{i \lambda k_{z}}{k}, & \gamma=\sqrt{\frac{9}{4}-\lambda^{2}-\mu^{2}}, & \ell=e B \tau^{2} .
\end{array}
$$

where $m$ and $e$ are the mass and charge of the complex scalar field, respectively. In terms of the Bogoliubov coefficient the pair production rate $\Gamma$ is given by [5]

$$
\Gamma=\frac{1}{\Omega^{4}(\tau) T L_{y}} \sum_{n=0}^{\infty} \int \frac{d k_{z}}{(2 \pi)} \frac{d k_{x}}{(2 \pi)}\left|\beta\left(k_{z}, n\right)\right|^{2},
$$

where $T$ is the time interval of the pair production and $L_{y}$ is the auxiliary parameter determining the normalization volume. Since the Bogoliubov coefficient $\beta$ is independent of the momentum $k_{x}$, the integral gives $e B L_{y} /(2 \pi)$ from the position of the center of the wave packet. Under the semiclassical condition $[2,3]$

$$
\lambda^{2}+\mu^{2} \gg 1
$$

the time when most of the particles are created, estimates $\tau k_{z} \sim-|\gamma|[2,3]$, hence the $k_{z}$ integral can be transformed into a $\tau$ integral. We then obtain

$$
\Gamma=\left(\frac{e B \Omega^{-2}}{2 \pi}\right)\left(\frac{H^{2}|\gamma|}{2 \pi}\right) \sum_{n=0}^{\infty}\left[\frac{e^{2 \pi|\kappa|}-1}{e^{2 \pi|\gamma|}-e^{-2 \pi|\gamma|}}+\frac{1}{e^{2 \pi|\gamma|}-1}\right],
$$

where

$$
|\kappa|=\frac{\lambda|\gamma|}{\sqrt{|\gamma|^{2}+(2 n+1) \ell}} .
$$

In fig. 1a the pair production rate $\Gamma$ given by eq. (7) is plotted as a function of the electric field for different intensities of the magnetic field. This figure illustrates that in the strong electric field regime 


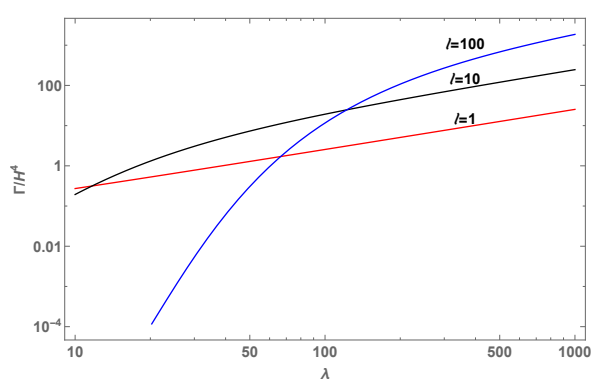

(a) For different values of $\ell$, the normalized pair production rate $\Gamma / H^{4}$ is plotted as a function of $\lambda$, in the lowest Landau state $n=0$ with $\mu=1$.

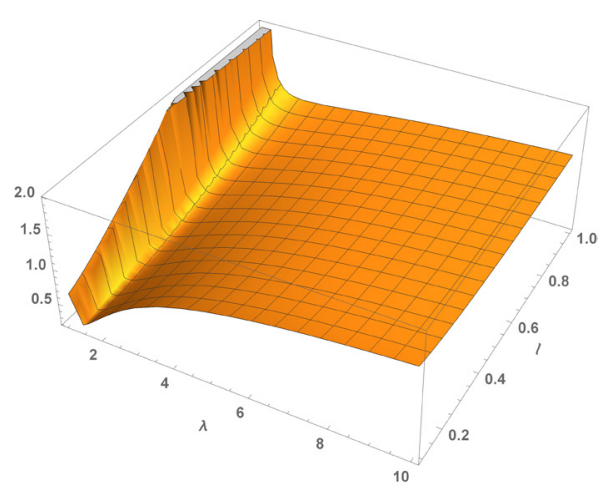

(b) The ratio of the induced current to the semiclassical current $J / J_{\text {sem }}$ is plotted as a function of $\lambda$ and $\ell$, in the lowest Landau state $n=0$ with $\mu=1$.

Figure 1

$\lambda \gg \max (1, \mu, \ell)$, the pair production rate is significant: $\Gamma \gg 1$. The first term in the square bracket in eq. (7) is the pair production rate from the electromagnetic field while the second term is the dS radiation weighted by the density of states for the electromagnetic field. Since the second term in $\Gamma$ (see e.q. (7)) is independent of Landau levels, its sum apparently diverges. However, the Riemann zeta function prescription of $\zeta(0)=-1 / 2$ together with the $n=0$ term gives a constant factor of $1 / 2$. Thus, the pair production from the zeta regularization technique leads to a finite result

$$
\Gamma=\left(\frac{e B \Omega^{-2}}{2 \pi}\right)\left(\frac{H^{2}|\gamma|}{2 \pi}\right)\left(\frac{1}{e^{4 \pi|\gamma|}-1}\right)\left[\frac{1}{2}+\sum_{n=0}^{\infty} e^{2 \pi(|k|+|\gamma|)}\right] .
$$

In the limit of zero electric field $E=0$, the first term in the square bracket of eq. (7) vanishes and the second term is the $\mathrm{dS}$ radiation weighted by the density of states of the magnetic field background. Therefore the gravitational pair production rate in $\mathrm{dS}$ is enhanced by the magnetic field. One can verify that the pair production expression given by eq. (7) leads to the well known Schwinger formula in the flat spacetime limit $(H=0)$.

\section{Induced Current}

Once the pair production rate $\Gamma$ is calculated, one can read the number density of the Schwinger pairs as $\mathcal{N} \sim \Gamma / H$. Then, the semiclassical conductive current $J_{\text {sem }}$ of the pairs having a charge $e$ and a velocity $v$ due to the background electric field is given by $J_{\text {sem }}=2 e \mathcal{N} v$. In this section we investigate numerically the expectation value of the current operator (which is referred to as the induced current) in the Hadamard in-state. The only nonvanishing component of the induced current, which is parallel to the electric field background, is given by [5]

$$
J=\frac{e H^{3} \ell}{4 \pi^{2}} \sum_{n=0}^{\infty} \int_{-\infty}^{+\infty} \frac{d k_{z}}{k}\left(\lambda-k_{z} \tau\right) e^{i \pi \kappa}\left|W_{\kappa, \gamma}(2 i k \tau)\right|^{2},
$$



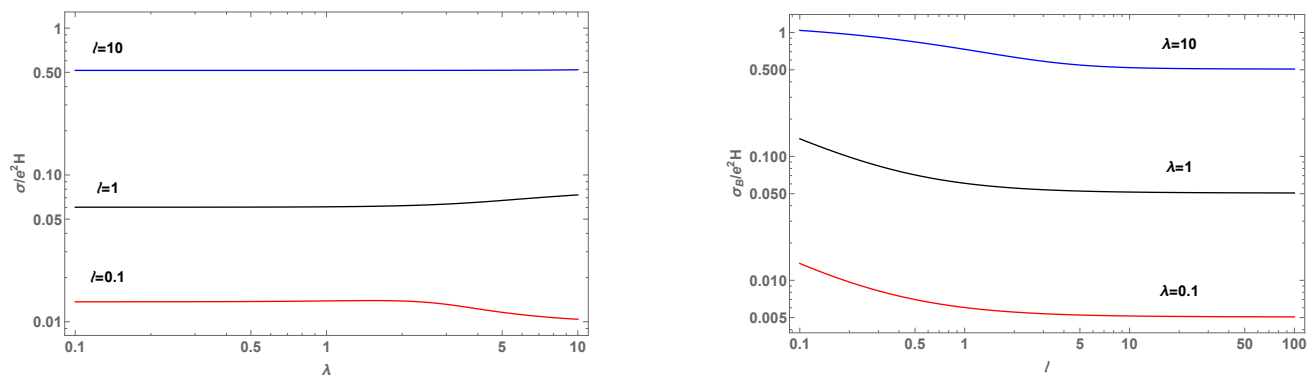

Figure 2: Left (right) panel: for different values of $\ell(\lambda)$, the normalized conductivity $\sigma / e^{2} H\left(\sigma_{B} / e^{2} H\right)$ is plotted as a function of $\lambda(\ell)$, in the lowest Landau state $n=0$ with $\mu=1$.

where $W$ is the Whittaker function. In the regime of $\lambda \gg 1$ the semiclassical condition (6) is satisfied, and the induced current (10) is comparable to the semiclassical current $J_{\text {sem }}$. Considering the ultrarelativistic particles with velocity $v \sim 1$, fig. 1b shows that the induced current $J$ approaches the semiclassical current $J_{\text {sem }}$ in the strong electric field regime $\lambda \gg \max (1, \mu, \ell)$. For a better understanding of the physics of the results, it is convenient to define the electric and magnetic conductivity $\sigma$ and $\sigma_{B}$ as

$$
\sigma=\frac{J}{E}, \quad \sigma_{B}=\frac{J}{\Omega^{-2} B} .
$$

In fig. 2 we plot $\sigma$ and $\sigma_{B}$ as a function of the electric and magnetic fields, respectively. The figure reveals that the induced current of a massive scalar field responds to the strong electromagnetic field as $J \propto B \cdot E$. In the infrared regime where the relations $\ell \ll \mu \ll \lambda \ll 1$ are satisfied, fig. 3 illustrates that the conductivity increases as the electric field decreases. This phenomena was first reported in [2] and dubbed infrared hyperconductivity.

\section{Conclusion}

We have reported here some results of [5]: the effect of a constant magnetic field on Schwinger mechanism in dS. We have described its effects and investigated some various limiting cases. These considerations are relevant to magnetogenesis scenarios. Using the zeta function regularization, we have obtained a finite expression for the Schwinger pair production rate. We have found that the Schwinger effect is enhanced by the magnetic field background. In the zero electric field limit, our result agrees with the Gibbons-Hawking radiation modulo the density of states for magnetic field. In addition, we have numerically examined the induced current. We have found that in the strong electromagnetic field the induced current is dominated by a term proportional to $E \cdot B$ and is thus approximately proportional to the pseudo-scalar of the Maxwell theory, which corresponds to the chiral magnetic effect for spin-1/2 fermions [9]. In the infrared regime, the current and conductivity are increasing as the electric field decreases, which leads to the infrared hyperconductivity phenomenon.

\section{Acknowledgement}

S. P. K. would like to thank Remo Ruffini at ICRANet, where this work was initiated and also WY. Pauchy Hwang for the warm hospitality at National Taiwan University. The work of S. P. K. was 

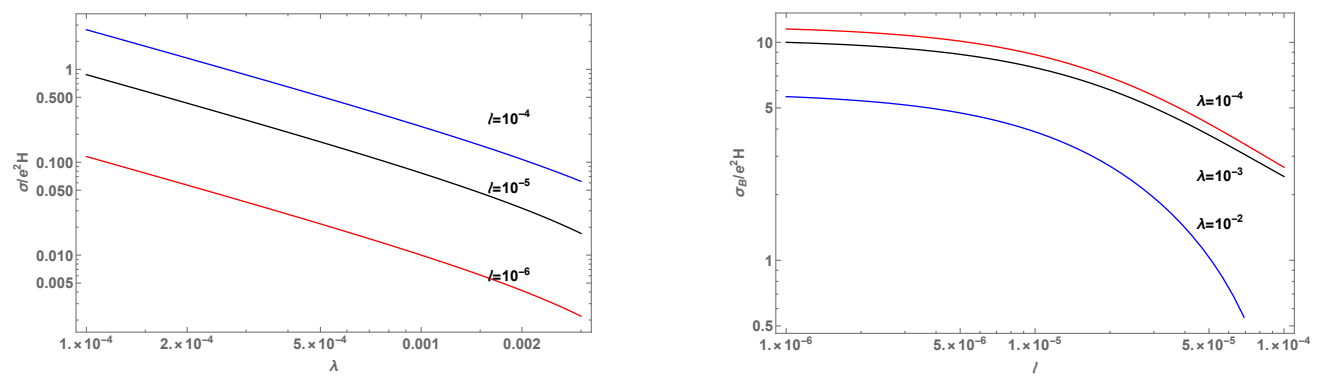

Figure 3: Left (right) panel: in the infrared regime, for different values of $\ell(\lambda)$, the normalized conductivity $\sigma / e^{2} H\left(\sigma_{B} / e^{2} H\right)$ is plotted as a function of $\lambda(\ell)$, in the lowest Landau state $n=0$ with $\mu=10^{-4}$.

supported by IBS (Institute for Basic Science) under IBS-R012-D1 and also by the Basic Science Research Program through the National Research Foundation of Korea (NRF) funded by the Ministry of Education (NRF-2015R1D1A1A01060626). E. B. and S.-S. X. would like to thank S. P. K. for the warm hospitality at Kunsan National University. E. B. is supported by the University of Kashan Grant No. $317203 / 2$.

\section{References}

[1] J. S. Schwinger, Phys. Rev. 82, 664 (1951).

[2] M. B. Fröb, J. Garriga, S. Kanno, M. Sasaki, J. Soda, T. Tanaka and A. Vilenkin, JCAP 1404, 009 (2014).

[3] T. Kobayashi and N. Afshordi, JHEP 1410, 166 (2014).

[4] E. Bavarsad, C. Stahl and S. S. Xue, Phys. Rev. D 94, no. 10, 104011 (2016).

[5] E. Bavarsad, S. P. Kim, C. Stahl and S. S. Xue, [arXiv:1707.03975 [hep-th]].

[6] K. Subramanian, Rept. Prog. Phys. 79, no. 7, 076901 (2016).

[7] S. Moradi, Mod. Phys. Lett. A 24, 1129 (2009); S. Haouat and R. Chekireb, Eur. Phys. J. C 72, 2034 (2012).

[8] C. Crucean and M. A. Băloi, Phys. Rev. D 93, no. 4, 044070 (2016).

[9] K. Fukushima, D. E. Kharzeev and H. J. Warringa, Phys. Rev. D 78, 074033 (2008). 